\title{
A Design Consideration for Planar Inverted Fractal Antenna to Minimize Length-Dependent Specific Absorption Rate
}

\author{
Akramul Haque, Sheikh Alimur Razi, Nur Mohammad, Md. Shamsul Arifin, \\ Quazi Delwar Hossain \\ Department of Electrical and Electronic Engineering, Chittagong University of Engineering and Technology (CUET), \\ Chittagong-4349, Bangladesh
}

\begin{tabular}{l} 
Article Info \\
\hline Article history: \\
Received Jul 1, 2018 \\
Revised Aug 21, 2018 \\
Accepted Sep 21, 2018 \\
\hline Keywords: \\
Characteristics impedance \\
PIFA antenna \\
Radiation minimization \\
Reflection co-efficient \\
Specific absorption rate
\end{tabular}

\begin{abstract}
This paper presents a numerical solution to minimize electromagnetic radiation from a Planar Inverted Fractal Antenna (PIFA) used in cellular phone. The PIFA is simulated using a semiconductor substrate having a dielectric constant of 3.38. The height of the dielectric substrate is $0.813 \mathrm{~mm}$. The designed antenna is simulated at a broad range of microwave frequency spectrum used in cellular communication. A $50-\mathrm{ohm}$ probe of $0.5 \mathrm{~mm}$ radius perpendicular to the ground substrate plate is used as a feeding medium. The antenna performance is evaluated for three different lengths keeping all other parameters constant. Simulation results show that the intended PIFA having a length of $20 \mathrm{~mm}$ can be used effectively to reduce the Specific Absorption Rate (SAR) of radiation. Moreover, the reflection coefficient was found to be minimal 0.1569 at $20 \mathrm{~mm}$ antenna length which is determined by characteristic impedance relation. Therefore, this investigation of minimizing the radiation absorption can be considered during the implementation phase of various cellular antennas to avoid radiation-related health hazards.
\end{abstract}

Copyright $(2018$ Institute of Advanced Engineering and Science. All rights reserved.

\section{Corresponding Author:}

Nur Mohammad,

Department of Electrical and Electronic Engineering,

Chittagong University of Engineering and Technology (CUET),

Chittagong-4349, Bangladesh.

Email: nur.mohammad@cuet.ac.bd

\section{INTRODUCTION}

The Electro Magnetic Radiation (EMR), in the long run, deteriorates human and plants health if the radiation exceeds a threshold level. Hence, it is required to control the EMR pollution to ensure health safety [1]. The EMR propagates as electric and magnetic fields through any medium. According to [2], the EMR pollution triggers the drop in the population growth rate of sparrows and bees, in exchange of which Colony Collapse Disorder (CCD) begins. It has been reported that under the EMR exposed environment, bats and sparrows show a disinclination in their behaviour. For example, in the USA, deadly crashes of birds with communication masts cause the extinction of millions of birds each year [2]. Furthermore, the EMR hampers brain activities, causes headaches, triggers memory loss as well as can cause cancer in some cases. It may initiate sensitive problems like congenital disability and low sperm count [3]. The EMR absorption depends on forms and dimensions of the organ, dielectric properties of respective body tissue. The absorption amount is indexed by the term specific absorption rate (SAR). The SAR value for a radio device is different for different body parts. The SAR is defined as the power, W absorbed due to exposure to an EMR by per kg of body tissue [4]. It depends on the electric field intensity, frequency of supply, polarisation of the field (far and near field) characteristics. Again, not only the fields that are emitting through the mobile is responsible for the SAR, but also reflected and scattered EMR from peripheral devices contribute to a high value of the SAR [5]. Regarding this, an investigation was conducted by Pack and Lee, who figured out the SAR values 
for several sizes and shapes of head. They examined by using finite difference time domain method for a broad range of frequency ranging from 835 to $1,765 \mathrm{MHz}$. [6].

In various research, authors found the sources of EMR and corresponding health issues [7-11]. Akihiro Tateno and Kensuke in their study found that mobile phones and tablet computers are two of the prime sources of the EMR pollution [10]. The power level of the EMR source and the distance between the source and receiver are the two significant variables that define the power density at a specific spot. The consistent growth of the mobile company as well as its user, the EMR pollution has indeed become a significant concern for humanity and the environment.

To minimise the adverse effects of the radiation, scientists and researchers are proposing different techniques and methods. In [7], Jalal J. Hamad Amin presented a resolution to implement optical fibre communication, replacing current microwave communication, which is costly for developing countries. The author also shared some tips and tricks for using cell-phone, which can reduce the probable detrimental effects of the EMR, however, ignored to way out to reduce the amount of radiated EMR. Yusuf Abdulrahman Sambo and et al. in [9] investigated multiple aspects including SAR shielding, Beam forming processes along with the installation of massive MIMO that can lessen the amount of radiation.

Recently, a Planar Inverted Fractal Antenna (PIFA) is reported in the literature to control the EMR level [12]. The PIFA is a sort of linear Inverted F-Antenna, a monopole antenna used in wireless communication [13]. It is manufactured by setting a metallic patch over a bed of dielectric, which is supported on a ground plate. The feeding method of the metallic patch is done either by adding microstrip transmission line or by a probe. Easy fabrication, low cost and small weigh are the reasons behind the massive using scenario of PIFA in the mobile industry.

Researchers are investigating ways to reduce the amount of EMR radiation from the cellular phones by incorporating new design strategies of the PIFA. Some recent studies revealed that the detrimental impacts of EMR radiation from the PIFA could be minimised by reducing the SAR [14-16]. In [8], Sang-il Kwak and et al. showed a process of designing PIFA with the artificial magnetic conductor. The SAR reduction mechanism was introduced considering a wideband code division multiple access bands, and a 43\% SAR reduction was obtained at the centre frequency. The same authors in their earlier publication [11], proposed two types of electromagnetic band gape model for SAR reduction of the EMR radiation. However, both the studies disregarded the effect of antenna length on the SAR reduction. To fill this gap, this paper proposes a PIFA, and its length is calibrated to find the dependence of SAR on the length. The SAR reduction empirical method is discussed, and the SAR values are measured for three different PIFA length, and the obtained results are compared.

Some of the other variables such as shorting pin width, probe feed radius, the distance between the shorting pin and the probe, the substrate material and the insulator height have less significance on the SAR [17]. Thereby impact of these variables is disregarded in this study.

In the next section methodology for this research is outlined followed by the results and discussion in Section 3. The concluding remarks are provided in Section 4 at the end.

\section{METHODOLOGY}

Table 1 enlists frequency band used in cellular communication. As seen in the table a broad spectrum of the high-frequency electromagnetic wave are used for different generation of communication technology [18]. Higher the frequency, the more the probability of energy being absorbed by body tissue. As the frequency of radiation increase, its ionization power also increases. Cell phone antennas are designed to transmit data at various frequencies depending on the communication generation.

Table 1 . The frequency range of various telecom generation.

\begin{tabular}{cl}
\hline Generation & \multicolumn{1}{c}{ Frequency Range } \\
\hline $2 \mathrm{G}$ & $869-890 \mathrm{MHz}$ (CDMA), $935-960 \mathrm{MHz}$ (GSM900), 1805-1880 MHz (GSM1800) \\
$3 \mathrm{G}$ & $2110-2170 \mathrm{MHz}$ \\
$4 \mathrm{G}$ & $800 \mathrm{MHz}, 2100-2600 \mathrm{MHz}$ \\
\hline
\end{tabular}

Due to resource limitation, the work is carried out by the Advanced Design System (ADS) simulation platform, because of its accuracy and easy-to-use interface. Figure 1 shows the diagram of a PIFA where L1 and L2 denote the length and width of the PIFA respectively, W is the width of the shorting pins, $\mathrm{D}$ is for the separation of feed and shorting pin, and $\mathrm{h}$ is the distance of PIFA above the ground plane. The antenna height, $\mathrm{h}$ is filled with an air substrate $(\varepsilon r=1.0)$. The shorting plate consists of a vertical conducting 
strip, and it is used not only to connect between the patch and ground but also to support the whole antenna. The $50 \Omega$ coaxial probe has a radius of $0.5 \mathrm{~mm}$ and is fed in the centre line of the rectangular patch.

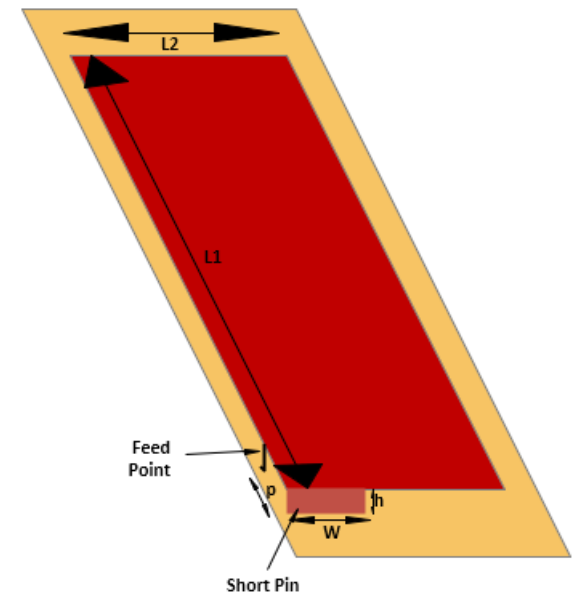

Figure 1. Planer inverted $\mathrm{F}$ antenna with a shorting plate

To reduce the SAR, the PIFA length L1 has been modified. Hence, this paper recommends the method of length reduction to minimise SAR. The designed PIFA has been printed over a Rogers' board and fed by a 50 -ohm probe of $0.5 \mathrm{~mm}$ radius. The probe was perpendicular to the ground plate. Table 2 shows the design specifications of the PIFA. The Rogers board indicated in ADS as RO4003C has a dielectric constant, $\varepsilon$ of 3.38. The thickness of the copper plate is $0.5 \mathrm{~mm}$. The area of the antenna is $\mathrm{L} 1 \times \mathrm{L} 2$, and the area of the ground plate is $\mathrm{Lg} \times \mathrm{Wg}$. The air $(\varepsilon=1.00)$ is considered as the insulation layer. A shorting strip connects the patch antenna to the ground as well as supports the whole antenna.

Table 2. Proposed PIFA antenna specifications

\begin{tabular}{cll}
\hline SL. No & Parameters & Specifications \\
\hline 1. & Shape & Rectangular \\
2. & Frequency of operation & $1.39-1.5 \mathrm{GHz}$ \\
3. & Dielectric constant of the substrate & 3.38 (Rogers RO4003C) \\
4. & Height of dielectric substrate & $0.813 \mathrm{~mm}$ \\
5. & Feeding Method & probe feed \\
\hline
\end{tabular}

The operating frequency of the PIFA can be roughly determined by the following set of equations (1-3) [17].

$$
\begin{aligned}
& L_{1}=\frac{\lambda_{d}}{4}=\frac{1}{4} \times \frac{c}{f \sqrt{\varepsilon_{r}}} \\
& L_{2}=\frac{c}{4 f} \sqrt{\frac{2}{\varepsilon_{r}+1}} \\
& f=\frac{c}{4\left(L_{1}+L_{2}\right)}
\end{aligned}
$$

Here, $\lambda \mathrm{d}$ is the wavelength inside the substrate, $\varepsilon \mathrm{r}$ is the relative permittivity, L1 and L2 are the length and width of the PIFA respectively, and c is the velocity of the EM wave.

The following set of equations provides the detail investigation on the SAR for antennas used in Wireless Communication [19]. The SAR has an inverse proportional relation with the return loss which is defined as the logarithm of the ratio of the incident power, Pi to the reflected power, Pr as shown in Equation (4).

$$
R L(d B)=10 \log _{10} \frac{P_{i}}{P_{r}}
$$


The lesser the reflected power the higher is the return loss, and the lower is the SAR. The reflection coefficient also carries excellent information about the SAR value. It is the ratio of the reflected voltage to the incident voltage, which is also related to the antenna impedance $\mathrm{ZL}$ and characteristics impedance Zo as given by Equation (5).

$$
|\Gamma|=\frac{V_{\text {reflected }}}{V_{\text {incident }}}=\frac{Z_{L}-Z_{0}}{Z_{L}+Z_{0}}
$$

The SAR in terms of the electrical conductivity $(\mathrm{S} / \mathrm{m})$ of tissue $(\sigma)$, the density of tissue in $\mathrm{Kg} / \mathrm{m} 3$ $(\rho)$ and the intensity of the electric field (E) is presented by Equation (6).

$$
\mathrm{SAR}=\frac{\sigma|E|^{2}}{2 \rho}
$$

The normalized SAR is given by Eq. (7).

$$
S A R_{\text {norm. }}=\frac{S A R_{\text {original }}}{\left(1-|\Gamma|^{2}\right)\left(1-\eta_{\text {rad. }}^{2}\right)}
$$

Here, SARoriginal means the SAR value that is calculated using Equation (6) moreover, nrad is the radiation efficiency.

\section{RESULTS AND DISCUSSION}

This section presents the simulation results to analyse the performance of the designed PIFA. The length $\mathrm{L} 1$ is changed from $30 \mathrm{~mm}$ to $20 \mathrm{~mm}$ with a $5 \mathrm{~mm}$ equal decrement keeping the width $\mathrm{L} 2$ and other parameters constant. Several test trials have been performed to achieve an enhanced frequency match at each step. Shortening the patch antenna to the ground helps to match the impedance. However, it makes the radiation impedance reactive. Thereby, a capacitive top loading is used to minimise this effect. Figure 2 shows the $2 \mathrm{D}$ and $3 \mathrm{D}$ view of the designed PIFA antenna for $30 \mathrm{~mm}$ length. For brevity, similar views for other two lengths have not been provided in this paper.

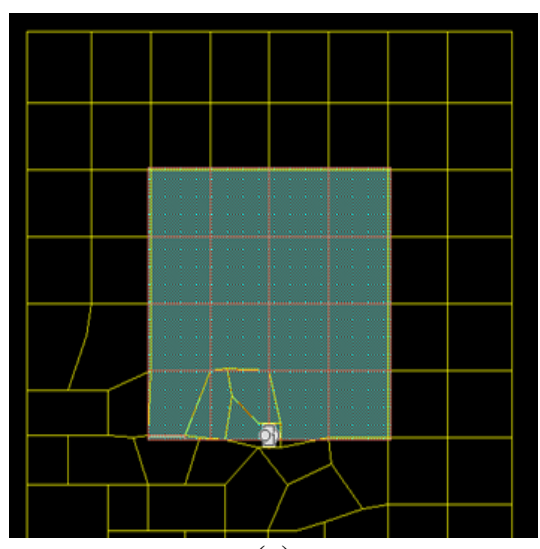

(a)

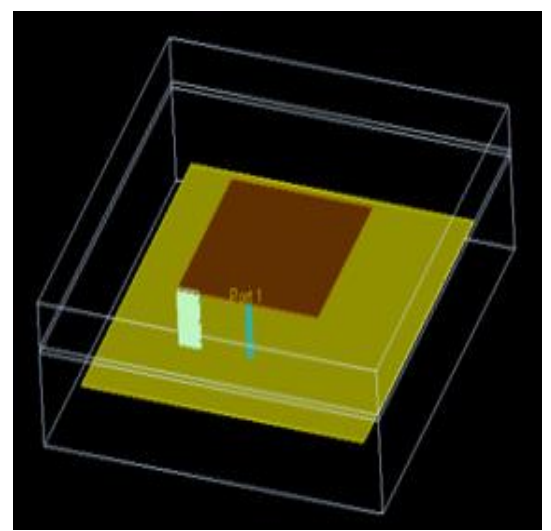

(b)

Figure 2. The designed PIFA antenna for $\mathrm{L} 1=30 \mathrm{~mm}$, (a) $2 \mathrm{D}$ view, (b) $3 \mathrm{D}$ view 


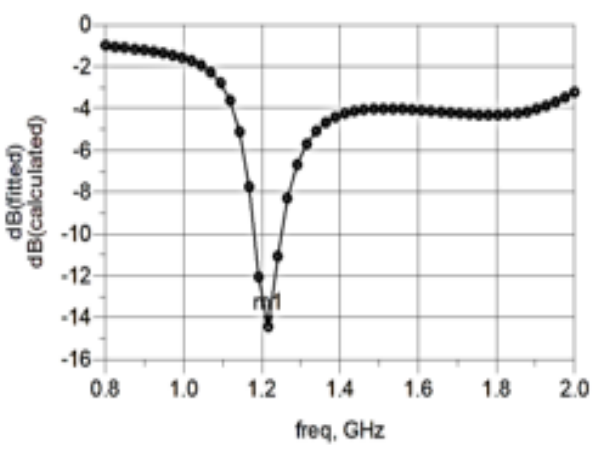

(a)

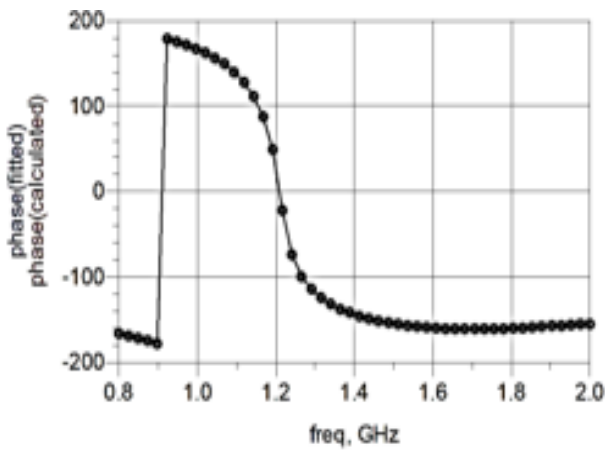

(b)

Figure 3. The return loss profile of PIFA antenna for $L_{l}=30 \mathrm{~mm}$ (a) Magnitude, and (b) phase angle spectrum

Figure 3 presents the simulation result. The magnitude and the phase angle spectrums of return loss for a broad range of frequency are shown in Figure 3(a) and 3(b) respectively. The actual magnitude of the return loss is the negative of the $\mathrm{Y}$-axis values of the magnitude spectrum. As shown in Figure 3(a), the magnitude of the return loss is found the maximum of $14.436 \mathrm{~dB}$ at $1.216 \mathrm{GHz}$. Figure 3 (b) shows the corresponding the phase shift of around -20 degree. The line impedance ZL is calculated from the Smith chart shown in Figure 4. The reflection coefficient is calculated to be $|\Gamma|=0.1753$ using Equation (5).

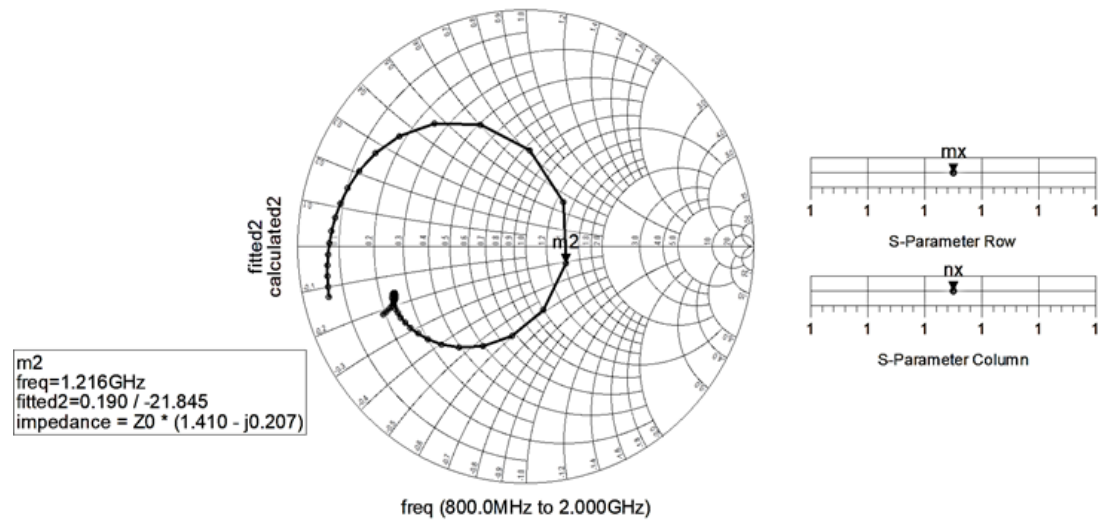

Figure 4. The Smith chart of PIFA antenna for L1 $=30 \mathrm{~mm}$

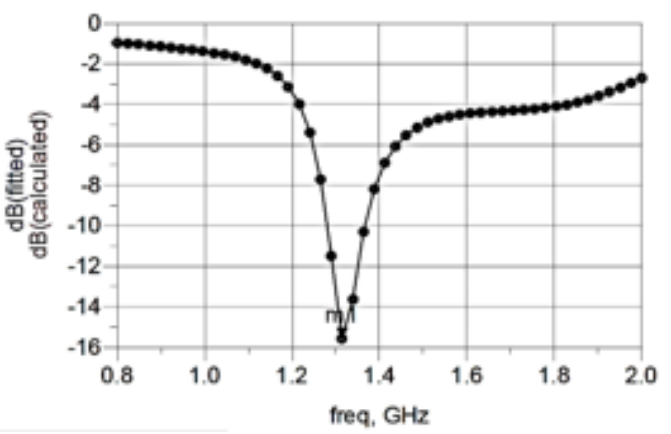

(a)

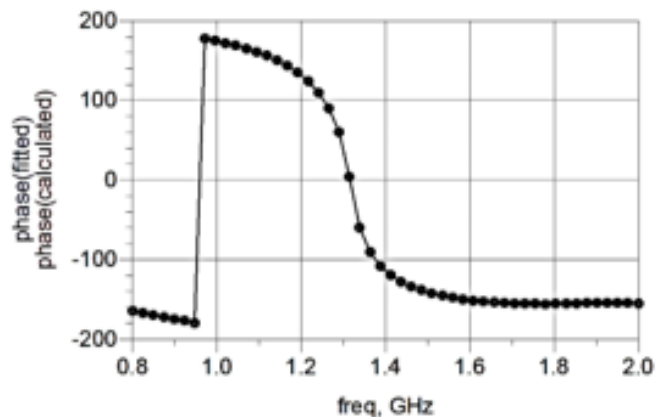

(b)

Figure 5. The return loss profile of PIFA antenna for $L_{l}=25 \mathrm{~mm}$ (a) Magnitude, and (b) phase angle spectrum 
The magnitude and phase angle spectrums of return loss for the $25 \mathrm{~mm}$ length of the PIFA are shown in Figure 5. As shown in Figure 5(a), the magnitude of the return loss is found the maximum of $15.566 \mathrm{~dB}$ at $1.314 \mathrm{GHz}$. Figure 5(b) shows the corresponding phase shift of around zero degrees. The line impedance $\mathrm{ZL}$ is obtained from the Smith chart shown in Figure 6, and the corresponding reflection coefficient is found to be $|\Gamma|=0.1661$.

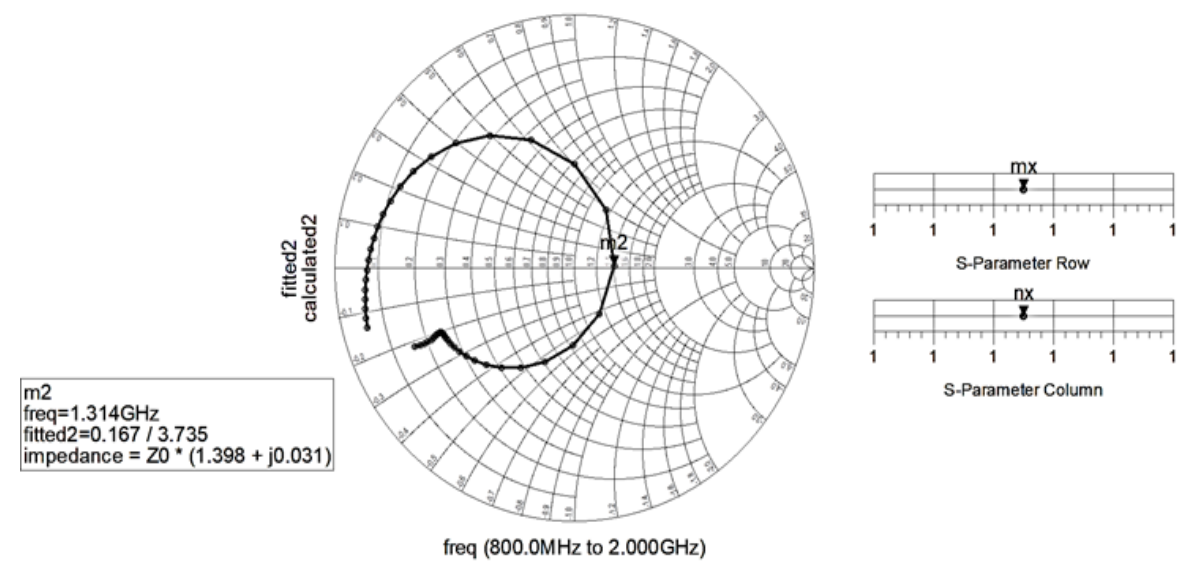

Figure 6.The Smith chart of PIFA antenna for L1 = $25 \mathrm{~mm}$

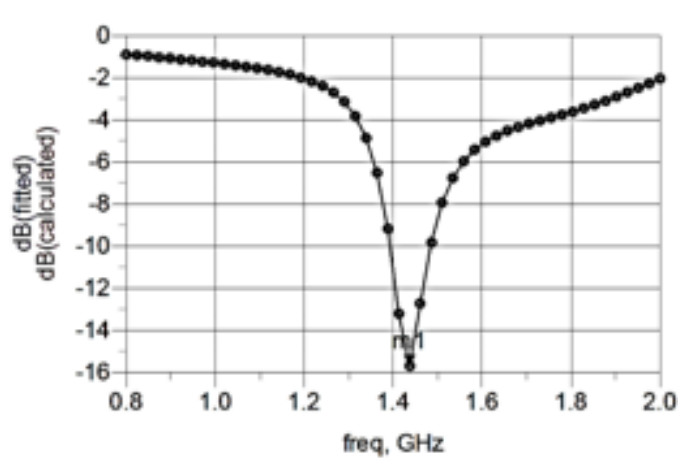

(a)

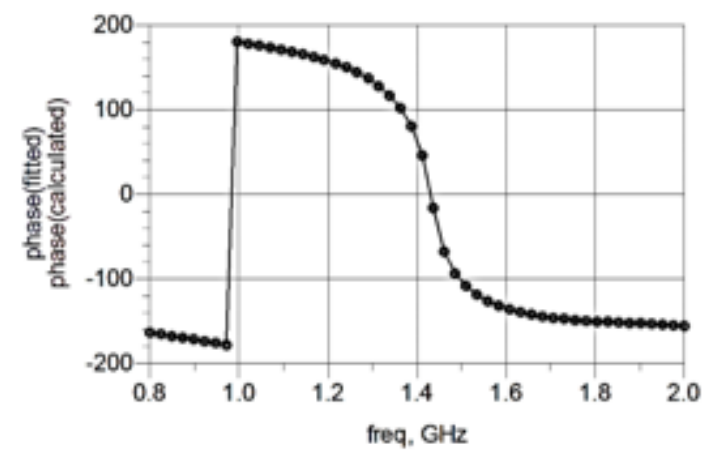

(b)

Figure 7. The return loss profile of PIFA antenna for $L_{l}=20 \mathrm{~mm}$ (a) Magnitude, and (b) phase angle spectrum

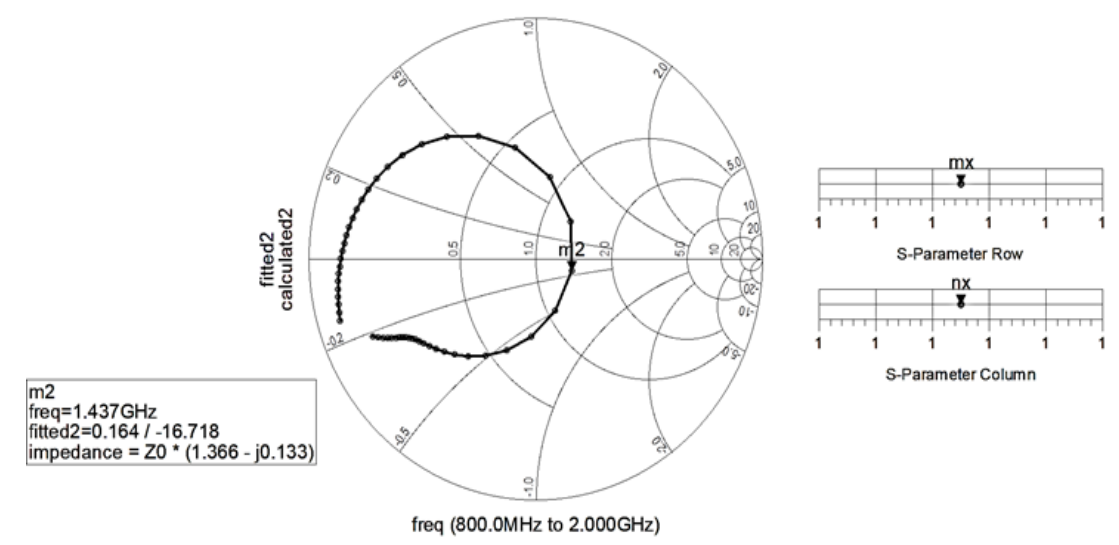

Figure 8. The Smith chart of PIFA antenna for L1 = $20 \mathrm{~mm}$ 
Similarly, the magnitude and phase angle spectrums of the return loss for the $20 \mathrm{~mm}$ length of the PIFA are shown in Figure 7. According to the Figure 7(a), the magnitude of the return loss is found the maximum of $15.686 \mathrm{~dB}$ at $1.437 \mathrm{GHz}$. Figure 7(b) shows the corresponding phase shift of around -20 degree. The line impedance ZL is obtained from the Smith chart shown in Figure 8, and the corresponding reflection coefficient is found to be $|\Gamma|=0.1569$.

In summary, the return loss is found the maximum, and the reflection coefficient is found minimum when the antenna length is $20 \mathrm{~mm}$.

The measurement of antenna impedance by varying the length of PIFA is listed in Table 3. As seen in the table, the antenna impedance approaches to probe impedance as the antenna length decreases providing a better impedance matching at $20 \mathrm{~mm}$. This signifies the robustness of the designed PIFA for the length above.

Table 3. Measurement of antenna impedance by varying the length of PIFA

\begin{tabular}{ccc}
\hline & $\begin{array}{c}\text { Length of PIFA } \\
\text { antenna }(\mathrm{mm})\end{array}$ & $\begin{array}{c}\text { Antenna } \\
\text { Impedance }\left(Z_{L}\right)\end{array}$ \\
\hline 1 & 30 & 71.25 \\
2 & 25 & 69.92 \\
3 & 20 & 68.62 \\
\hline
\end{tabular}

In summary, the return loss increases as the antenna length L1 decreases. Improved return loss signifies the diminishing of reflected power according to the Equation (4). This means that, for a certain amount of received power, the required amount of incident power is reduced, which in turn lessens the amount of transmitted power. Thereby the electric field is reduced. Consequently, the SAR reduces according to Equation (6).

From another point of view, it is observed that the reflection coefficient decreases with the reduction in antenna length which implies that, the reflected voltage also reduces. As the reflected voltage reduces, the incident voltage requirement is reduced for a fixed amount of the induced voltage in the receiver antenna according to Equation (5). As a result, the electric field intensity of antenna decreases. As seen in Equation (6), for a set value of tissue conductivity and density, the SAR also decreases. Equation (7) shows that the normalized SAR diminishes as well.

Though the phase difference between incident and reflected waves changes, however, this does not carry on any significant impact on SAR value. The Smith Charts show that significant changes in the length do not affect the antenna impedance noticeably.

\section{CONCLUSION}

In this paper, we have analyzed the length dependent return loss, reflection coefficient and specific absorption rate of EMR by varying the PIFA length. The PIFA is designed and simulated in ADS platform. The study revealed that the return loss increases while the reflection coefficient decreases, as the antenna length, decreases. Also, a decrease in the antenna length yields a decrease in the SAR value.

The key limitation of this length reduction is that the working frequency of the antenna changes with decreasing length which should be taken into account during the design process. This problem can be minimized by two methods: one is to increase the air gap between the ground plate and antenna, and the second one is to change the material of insulation layer.

\section{REFERENCES}

[1]. Nehru V. "Global Wireless Spiderweb: The Invisible Threat Posed by Wireless Radiation". 1st ed. Gurgaon, India: Partridge Publishing, India, 2016.

[2]. Albert M. Manville II. "Impacts to Birds and Bats Due to Collisions and Electrocutions from Some Tall Structures in the United States: Wires, Towers, Turbines, and Solar Arrays-State of the Art in Addressing the Problems". In: Problematic Wildlife: A Cross-Disciplinary Approach. Angelici F. Arlington, VA, USA: Springer, Cham; 2016: 415-42. DOI: 10.1007/978-3-319-22246-2.

[3]. Anon. "EMF Health Effects"., 2018. [Online]. Available: https://www.safespaceprotection.com/emf-healthrisks/emf-health-effects/cell-towers/. [Accessed: Mar. 21, 2018 ].

[4]. Choi D, Shin C, Kim N, Shin H. "Design and SAR Analysis of Broadband PIFA with Triple Band". Progress In Electromagnetics Research Symposium. Hangzhou, China. PIERS, online 2005; 1: 290-3. DOI: 10.2529/PIERS041212211650.

[5]. Hoque AKMF, Hossain MS, Mollah AS, Akramuzzaman M. "A Study on Specific Absorption Rate (SAR) Due to Non-Ionizing Radiation from Wireless/Telecommunication in Bangladesh".American Journal of Physics and 
Applications. 2013; 1(3): 104-10. DOI: 10.11648/j.ajpa.20130103.18.

[6]. Lee A, Choi H, et al. "Effect of Head Size for Mobile Phone Exposure on EM Absorption".Asia-Pacific Microwave Conference (APMC). Taipei, Taiwan. IEEE.2001; 384-7. DOI: 10.1109/APMC.2001.985668.

[7]. Jalal J. Hamad Ameen. "Reduction of Cell Phone Electromagnetic Radiation Effect on Human Body". International Journal of Sciences. 2014; 3(March): 19-23.

[8]. Kwak S il, Sim D, Kwon JH, Yoon YJ. "Design of PIFA WithMetamaterials for Body-SAR Reduction inWearable Applications". IEEE Transactions on Electromagnetic Compatibility. 2017; 59(1): 297-300. DOI: 10.1109/TEMC.2016.2593493.

[9]. Sambo YA, Heliot F, Imran MA. "A survey and tutorial of electromagnetic radiation and reduction in mobile communication systems". IEEE Communications Surveys and Tutorials. 2015; 17(2): 790-802. DOI: 10.1109/COMST.2014.2364136.

[10]. Tateno A, Tanaka K, et al. "Comparison of SAR in Human Body Radiated from Mobile Phone and Tablet Computer". International Symposium on Electromagnetic Compatibility, Tokyo (EMC'14/Tokyo). Tokyo, Japan: IEEE. 2014; 186-9.

[11]. Kwak S, Sim DU, Kwon JH, Yun JH. "SAR reduction of PIFA with EBG structures for mobile applications". IEICE Transactions on Communications. 2009; E92-B(11): 3550-3. DOI: 10.1587/transcom.E92.B.3550.

[12]. Chattha HT, Huang Y, Ishfaq MK, Boyes SJ. "A Comprehensive Parametric Study of Planar Inverted-F Antenna". Wireless Engineering and Technology. 2012; 03(01): 1-11. DOI: 10.4236/wet.2012.31001.

[13]. Douglas H. Werner, Raj Mittra. "Fractal-Shaped Antennas". In: Frontiers in Electromagnetics. 1st ed. New Jersey, USA. Wiley-IEEE Press.2000: 814. DOI: 10.1109/9780470544686.ch2.

[14]. Yousaf J, Jung H, Kim K, Nah W. "Design, Analysis, and Equivalent Circuit Modeling of Dual-Band PIFA Using a Stub for Performance Enhancement". Journal of Electromagnetic Engineering and Science. 2016; 16(3): 169-81. DOI: 10.5515/JKIEES.2016.16.3.169.

[15]. Rathwa RR, Kotak NA, Tarpara N. "Design of a wideband Planar Inverted F-Antenna ( PIFA ) for Wireless Communication Devices". International Journal of Scientific Research in Science, Engineering and Technology. 2018; 4(2): 128-32.

[16]. Rana S, Thakur A, et al. "A wideband planar inverted F antenna for wireless communication devices". International Conference on Advances in Computing, Communication, \& Automation (ICACCA) (Spring). Dehradun, India: IEEE. 2016; DOI: 10.1109/ICACCA.2016.7578875.

[17]. S. Palanivel Ranjan, Dr R. Sukanesh, S. Vijayprasath. "Performance Evaluation of Mobile Phone Radiation Minimization through Characteristic Impedance Measurement for Health-care Applications". 2012 IEEE International Conference on Advanced Communication Control and Computing Technologies (ICACCCT).Ramanathapuram, India: IEEE. 2012; 1-4. DOI: 10.1109/ICACCCT.2012.6320722.

[18]. Wong KL, Lee GY, Chiou TW. "A low-profile planar monopole antenna for multiband operation of mobile handsets". IEEE Transactions on Antennas and Propagation. 2003; 51(1): 121-5. DOI: 10.1109/TAP.2003.809044.

[19]. Shi Y, Sun H, Liang C-H. "SAR Study of Antennas in Wireless Communication Terminals".Microwave and Optical Technology Letters. 2014; 56(10): 2361-5. DOI: 10.1002/mop. 